\title{
Quasiexcitons in Incompressible Quantum Liquids
}

\author{
Arkadiusz Wójs, ${ }^{1,2}$ Anna Gładysiewicz, ${ }^{2}$ and John J. Quinn ${ }^{1}$ \\ ${ }^{1}$ University of Tennessee, Knoxville, Tennessee 37996, USA \\ ${ }^{2}$ Wroclaw University of Technology, 50-370 Wroclaw, Poland
}

\begin{abstract}
Photoluminescence (PL) has been used to study two-dimensional incompressible electron liquids in high magnetic fields for nearly two decades. However, some of the observed anomalies coincident with the fractional quantum Hall effect are still unexplained. We show that emission in these systems occurs from fractionally charged "quasiexciton" states formed from trions correlated with the surrounding electrons. Their binding and recombination depend on the state of both the electron liquid and the involved trion, predicting discontinuities in PL and sensitivity to sample parameters.
\end{abstract}

PACS numbers: 71.35.Pq, 71.35.Ji, 71.10.Pm, 73.43.-f

The density of states of a two-dimensional electron gas (2DEG) in a high magnetic field $B$ consists of discrete Landau levels (LL's). When all electrons fall into the lowest LL, it is their mutual interaction that solely determines the ground state (GS) and low-energy excitations. Reminiscent of atomic physics except for the macroscopic LL degeneracy, this makes the 2DEG at high $B$ an ideal laboratory of many-body physics in extended systems.

The incompressible quantum liquids (IQL's) [1 were originally discovered in transport experiments [2] over two decades ago, but it took 15 years to demonstrate their hallmark fractionally charged quasiparticles (QP's) by shot-noise studies [3]. Photoluminescence (PL) was also used to probe IQL's, revealing anomalies at the LL fillings $\nu$ coincident with fractional quantum Hall effect [4, 5, 6, 7, 8] (usually doublets at $\nu \approx \frac{1}{3}$ or $\frac{2}{3}$, but other features, too $[9,10])$. Other optical experiments include PL with acceptor-bound holes 11] and Raman scattering 12.

The connection of PL anomalies with the microscopic properties of IQL's has been studied theoretically for over a decade. Depending on the strength and resolution of the Coulomb potential of photo-injected holes (controlled by $h-2$ DEG separation [7]), the observed doublets were attributed either to the "bare exciton" and "magnetoroton assisted" emission 13, 14] (efficient due to "gigantic suppression of the exciton dispersion by an IQL" 14|), or to recombination of different "anyon excitons" 15, 16 consisting of several fractional IQL QP's bound to a hole. However, understanding of all reported anomalies is not yet complete and, e.g., discontinuities reported in Refs. 5, 8] remain, to our best knowledge, unexplained.

To appreciate the complexity of the problem, one must recall that: (i) Even an unperturbed IQL has complicated dynamics whose understanding involves concepts of Laughlin correlations and fractionally charged QP's [1], anyon statistics 17], Haldane hierarchy [18], or composite fermions (CF's) 19]. (ii) Emergence of "multiplicative states" in $e-h$ fluids with "hidden symmetry" (HS) 20] greatly simplifies their optical response. (iii) Breaking of HS in real systems (due to finite layer widths $w$, charge separation, LL and valence band mixing, or disorder) re- stores the possibility of IQL-related anomalies in PL.

The HS-related effects in real quantum wells are well known in the "dilute" regime $(\nu \ll 1)$, in which PL is determined by recombination of excitons $(\mathrm{X}=e+h)$ and trions $\left(\mathrm{X}^{-}=2 e+h\right)[2,21]$. HS precludes radiative complexes larger than $\mathrm{X}$, allowing for only one trion "dark triplet" $\mathrm{X}_{\mathrm{t}}^{-} 22$. It is only due to the LL mixing that a "bright singlet" $\mathrm{X}_{\mathrm{s}}^{-}$occurs as well [23, 24].

In the "liquid" regime, few-body excitonic effects compete with many-body IQL dynamics, adding to each one's own complexity almost to guarantee fascinating physics. Different photo-excitations weakly coupled to the remaining IQL were proposed. In the anyon exciton model [15, 16] applicable for structures with strong charge separation (heterojunctions or wide asymmetric wells), the holes repel quasiholes (QH's) and attract quasielectrons (QE's) of the IQL. The "dressed exciton" concept [13, 14] introduced for narrower wells, involves the X's coupled to magnetorotons of the IQL. In another approach 16, 25 the $\mathrm{X}^{-}$'s correlate with the surrounding electrons.

In this Letter we develop the idea of trions immersed in a Laughlin IQL and predict discontinuity of the PL spectrum at $\nu=\frac{1}{3}[5,8]$. We show that trions remain stable in realistic doped wells, but acquire effective charge $\mathcal{Q}$ of up to one "Laughlin quantum" $\varepsilon=\frac{1}{3} e$ due to partial screening by the IQL. In analogy with $\mathrm{X}$ and $\mathrm{X}^{ \pm}$, we find neutral and charged "quasiexcitons" (QX's): $\mathcal{X}$ and $\mathcal{X}^{ \pm 1 / 3}$. They consist of a trion which is Laughlincorrelated with the IQL and binds 0,1 , or 2 QH's. The $\mathcal{X}^{ \pm 1 / 3}$ binding energies are directly observable in $\mathrm{PL}$, and their order-of-magnitude reduction from the $\mathrm{X}^{ \pm}$is an $i n$ dication of the fractional charge of their constituents.

For spin-polarized systems, we elucidate the earlier theory [13, 14] by identifying the "dressed exciton" with $\mathcal{X}$, its suppressed dispersion with the $\mathcal{X}^{-1 / 3}-\mathrm{QH}$ pseudopotential, and the "magnetoroton-assisted emission" with the $\mathcal{X}^{-1 / 3}$ recombination. The PL discontinuity proposed here due to charged QX's is a different effect, requiring no thermal activation and no charge separation.

In unpolarized systems, we find a spin-flip $\mathcal{X}$ whose steep dispersion prevents charging and removes the PL discontinuity. Competition between $\mathrm{X}_{\mathrm{s}}^{-}$and $\mathrm{X}_{\mathrm{t}}^{-}$in real- 
istic wells predicts dependence of PL anomalies on $w$.

We use exact numerical diagonalization for $N \leq 10$ electrons and one hole on a sphere 18] (with radius $R$, monopole strength $2 Q=4 \pi R^{2} B e / h c$, magnetic length $\lambda=R / \sqrt{Q}$ ). Interaction matrix elements were integrated taking subband wavefunctions $\phi_{e, h}(z)$ calculated self-consistently [26] for $w=10$ and $20 \mathrm{~nm}$ GaAs wells, doped on one side to $n=2 \cdot 10^{11} \mathrm{~cm}^{-2}$ (yielding $\nu=\frac{1}{3}$ at $B=25 \mathrm{~T}$, the values used further on). Finite size errors were minimized by extrapolation to $\lambda / R \rightarrow 0$.

We begin with calculation of $\mathrm{X}^{-}$Coulomb binding energies $\Delta$ using $\phi_{e, h}$, i.e., in mean normal electric field but ignoring in-plane $\mathrm{X}^{-}$-IQL coupling. We included five LL's and two $\phi$-subbands for both $e$ and (heavy) $h$, but neglected valence subband mixing. For $w=10 \mathrm{~nm}$, $\Delta_{\mathrm{s}}=2.3 \mathrm{meV}$ and $\Delta_{\mathrm{t}}=1.5 \mathrm{meV}$, in qualitative agreement with Refs. 23, 24], also predicting the $\mathrm{X}_{\mathrm{s}}^{-}$ground state (GS). For $w=20 \mathrm{~nm}$, neither symmetric-well nor lowest-subband approximation works well (e.g., the latter exaggerates charge separation in $\mathrm{X} / \mathrm{X}^{-}$and predicts breakup of $\mathrm{X}_{\mathrm{s}}^{-}$). Our best estimates, $\Delta_{\mathrm{s}}=1.5 \mathrm{meV}$ and $\Delta_{\mathrm{t}}=1.2 \mathrm{meV}$, are rather sensitive to the parameters, making prediction of the $\mathrm{X}^{-}$GS in real samples difficult and somewhat pointless. However, we expect that the $\mathrm{X}_{\mathrm{t}}^{-}$'s, additionally favored by the Zeeman energy, could at least coexist with the $\mathrm{X}_{\mathrm{S}}^{-}$'s at finite temperatures.

Let us immerse a trion $\left(\mathrm{X}_{\mathrm{s}}^{-}\right.$or $\mathrm{X}_{\mathrm{t}}^{-}$, depending on $w, n$, and $B)$ in an IQL. Effective $e-\mathrm{X}^{-}$pseudopotentials are similar 24] to the $e-e$ one 27]. In the lowest LL, this causes similar $e-e$ and $e^{-\mathrm{X}^{-}}$correlations, described in a generalized two-component [25] CF picture [19] by attachment of $2 p$ flux quanta to each $e$ and $\mathrm{X}^{-}$. At Laughlin/Jain fillings $\nu_{\mathrm{IQL}}=s /(2 p s+1)$, electrons converted to $\mathrm{CF}_{e}$ 's fill the lowest $s$ LL's in effective magnetic field $B^{*}=B-2 p n(h c / e)=B /(2 p s+1)$. At $\nu \neq \nu_{\mathrm{IQL}}$, QE's in the $(s+1)^{\text {st }}$ or QH's in the $s^{\text {th }} \mathrm{CF}_{e}$-LL occur, carrying effective charge $\varepsilon= \pm e /(2 p s+1)$. We find that, similarly, an $\mathrm{X}^{-}$is converted to a $\mathrm{CF}_{\mathrm{X}^{-}}$with charge $\mathcal{Q}=-\varepsilon$.

A trion coupled to an IQL and carrying reduced charge is a many-body excitation. To distinguish it from an isolated $2 e+h$ state, we call it a charged quasiexciton (QX) and denote by $\mathcal{X}^{-} \equiv \mathcal{X}^{-\varepsilon}$. Being negatively charged, an $\mathcal{X}^{-}$interacts with IQL QP's. At $\nu<\nu_{\mathrm{IQL}}$, the $\mathcal{X}^{-}$binds a $\mathrm{QH}$ to become a neutral $\mathcal{X}^{-} \mathrm{QH}=\mathcal{X}$, with a binding energy called $\Delta^{0}$. Depending on sample parameters and spin of the trion, $\mathcal{X}$ may bind an additional $\mathrm{QH}$ to form a positively charged $\mathcal{X}^{-} \mathrm{QH}_{2}=\mathcal{X}^{+}$, with binding energy $\Delta^{+}$. At $\nu>\nu_{\mathrm{IQL}}$, the $\mathcal{X}^{+}$attracts and annihilates a $\mathrm{QE}$ : $\mathcal{X}^{+}+\mathrm{QE} \rightarrow \mathcal{X}$; this process releases energy $\Delta_{\mathrm{IQL}}-\Delta^{+}$ (where $\Delta_{\mathrm{IQL}}=\mathcal{E}_{\mathrm{QE}}+\mathcal{E}_{\mathrm{QH}}$ is the IQL gap). The $\mathcal{X}$ may annihilate another $\mathrm{QE}: \mathcal{X}+\mathrm{QE} \rightarrow \mathcal{X}^{-}$, with energy gain

$$
\Delta^{-}=\Delta_{\mathrm{IQL}}-\Delta^{0}
$$

that can be interpreted as $\mathcal{X}^{-}$binding energy.

The $\mathcal{X}$ and $\mathcal{X}^{ \pm}$are different states in which a hole can exist in an IQL. If $\Delta^{ \pm}>0$, then depending on $\nu$, either

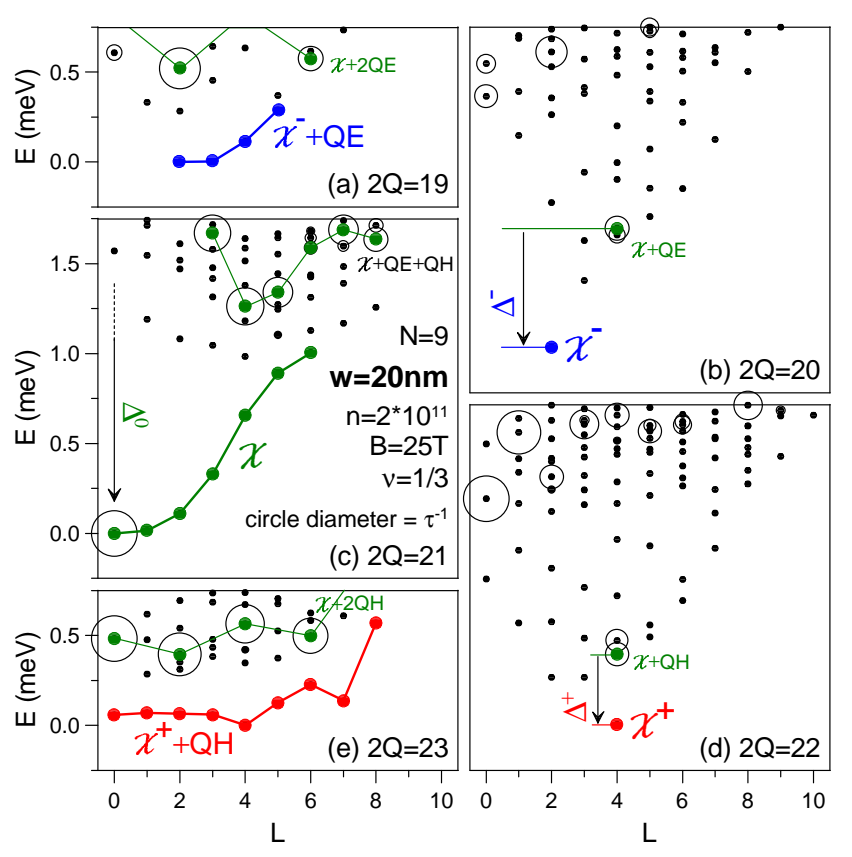

FIG. 1: (color online) Excitation energy spectra (energy $E$ as a function of total angular momentum $L$ ) of $9 e+h$ systems on a sphere, with up to two QE's or QH's in Laughlin $\nu=\frac{1}{3}$ IQL. Oscillator strengths $\tau^{-1}$ are indicated by open circles.

$\mathcal{X}^{-}$or $\mathcal{X}^{+}$is the most strongly bound state. If $\Delta^{-} \neq \Delta^{+}$, the PL spectrum will be discontinuous at $\nu_{\mathrm{IQL}}$. For longlived $\mathcal{X}^{ \pm}$(made of a dark $\mathrm{X}_{\mathrm{t}}^{-}$), recombination of the $\mathcal{X}$ is also possible, especially at $\nu \approx \nu_{\mathrm{IQL}}$ (within a Hall plateau), when QP localization impedes $\mathcal{X}^{ \pm}$formation.

The QX's resemble normal excitons in $n$ - or $p$-type systems, except that the concentration of their constituent QP's can be varied (in the same sample) by a magnetic field. Also, their kinetics $\left(\mathcal{X} \leftrightarrow \mathcal{X}^{ \pm}\right)$is more complicated because of the involved $\mathrm{QE}-\mathrm{QH}$ annihilation.

We have tested the QX idea numerically for Laughlin $\nu=\frac{1}{3}$ IQL. First, we calculated spin-polarized $\mathrm{Ne}+h$ energy spectra for $w=20 \mathrm{~nm}$, in search of the $\mathrm{QX}_{\mathrm{t}}$ 's. The $\mathrm{X}_{\mathrm{t}}^{-}$has $94 \%$ squared projection onto the lowest LL, so we ignored LL mixing in the $N e+h$ calculation (direct tests confirmed that it is negligible). The low-lying states in Fig. 1 are understood using the CF picture [19, 25] and addition rules for angular momentum. On a sphere, the $\mathrm{CF}$ transformation introduces an effective monopole strength $2 Q^{*}=2 Q-2(K-1)$, where $K=N-1$ is the total number of free electrons and $\mathrm{X}^{-}$'s. The angular momenta of constituent QP's are $l_{\mathrm{QH}}=Q^{*}, l_{\mathrm{QE}}=Q^{*}+1$, and $l_{\mathcal{X}^{-}}=Q^{*}-1$. The $\mathcal{X}^{-}$is a dark GS in (b) at $L=l_{\mathcal{X}^{-}}=2$, and $\mathcal{X}^{+}$is found in (d) at $L=l_{\mathcal{X}^{+}}=$ $\left|\left(2 l_{\mathrm{QH}}-1\right)-l_{\mathcal{X}^{-}}\right|=4$. Bands of $\mathcal{X}^{-}-\mathrm{QE}$ and $\mathcal{X}^{+}-\mathrm{QH}$ pairs are marked in (a) and (e). In (c) the radiative $L=0$ GS is a multiplicative state, opening a $\mathcal{X}=\mathcal{X}^{-}-\mathrm{QH}$ band 16], earlier called a "dressed exciton" and identified [13, 14] as responsible for the doublet structure in PL. 


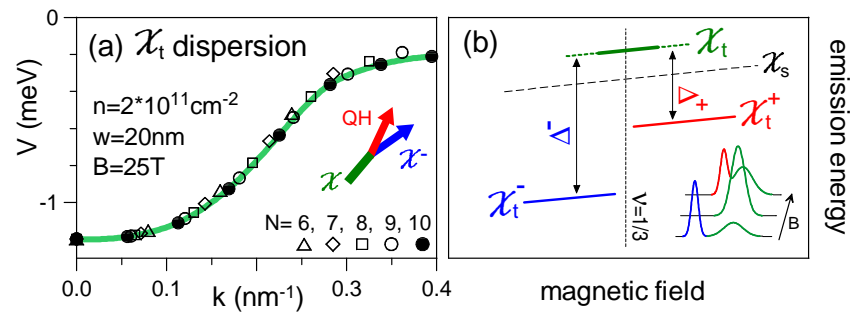

FIG. 2: (color online) (a) Dispersion of neutral quasiexciton $\mathcal{X}_{\mathrm{t}}$ in Laughlin $\nu=\frac{1}{3} \mathrm{IQL} ; \mathcal{X}_{\mathrm{t}}$ splits into $\mathcal{X}_{t}^{-}$and QH at $k>0$. (b) Schematic of PL discontinuity due to $\mathcal{X}_{\mathrm{t}}^{ \pm}$emission.

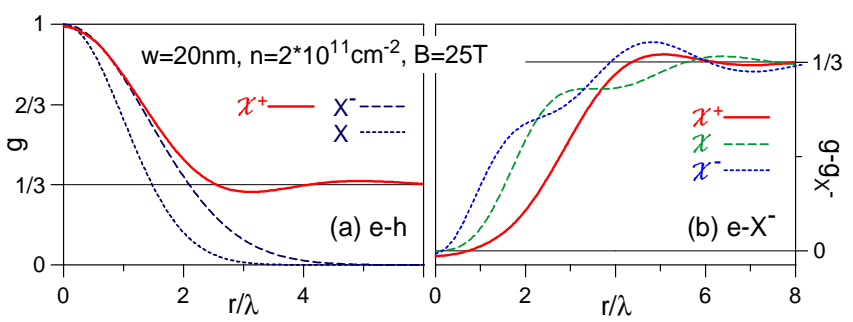

FIG. 3: (color online) (a) $e-h$ PDF's $g(r)$ of quasiexciton $\mathcal{X}_{\mathrm{t}}^{+}$ and isolated $\mathrm{X}_{\mathrm{t}}^{-}$and $\mathrm{X}$, normalized to measure local filling factor. (b) $e-\mathrm{X}^{-}$PDF's for different QX's; curve for $\mathcal{X}^{+}$ resembles $e-e \mathrm{PDF}$ of Laughlin liquid; shoulders for $\mathcal{X}$ and $\mathcal{X}^{+}$reflect additional charge quanta pushed onto the hole.

The continuous $\mathcal{X}$ dispersion shown in Fig. 2(a) results 13, 14 from the in-plane dipole moment being proportional to the wavevector $k=l / R$. Here we find why it is suppressed (compared to $\mathrm{X}$ ): because of the reduced charge of the $\mathcal{X}$ 's constituents, $\mathcal{X}_{t}^{-}$and $\mathrm{QH}$. In fact, the $\mathcal{X}$ and $\mathrm{X}$ dispersions become similar when energy and length scales are rescaled in account of the $e \rightarrow \varepsilon$ charge reduction. We also explain the emission from $\mathcal{X}$ at $k \lambda \sim 1.5$, proposed 13, 14] for the lower peak in PL, as the $\mathcal{X}^{-} \rightarrow$ QE recombination assisted by QH scattering. However, note that a small $d V / d k$ and a large $\tau^{-1}$ at $k \lambda \sim 1.5$ needed for this emission requires $w>20 \mathrm{~nm}$.

By identifying the multiplicative states containing an $\mathcal{X}$ with $k=0$, one can estimate $\Delta^{ \pm / 0}$ as marked in Fig. 1(b)-(d). More accurate values were obtained by comparing spectra in which $\mathcal{X}^{ \pm}, \mathcal{X}$, or $\mathrm{QP}$ is alone in the IQL, followed by extrapolation to $N \rightarrow \infty$ : $\mathcal{E}_{\mathrm{QH}}=0.73 \mathrm{meV}, \mathcal{E}_{\mathrm{QE}}=1.05 \mathrm{meV}, \Delta^{0}=1.20 \mathrm{meV}$, $\Delta^{-}=0.52 \mathrm{meV}$, and $\Delta^{+}=0.27 \mathrm{meV}$. Depending on $\mathcal{X}^{0} / \mathcal{X}^{ \pm}$kinetics, either $\Delta^{+} \neq \Delta^{-}$or $\Delta^{0} \neq \Delta^{ \pm}$asymmetry will make PL energy jump at $\nu=\frac{1}{3}$, as sketched in Fig. 2(b). Similar behavior has been observed [5, 8].

The $\mathcal{X}^{ \pm}$discontinuity is different from that due to anyon excitons [15, 16] anticipated in much wider wells. The two can be distinguished by different magnitude $\left(\sim \Delta_{\text {IQL }}\right.$ vs. $\left.\Delta^{ \pm}\right)$and opposite direction of the jump of emission energy when passing through $\nu=\frac{1}{3}$. In the present case, the small ratio of $\mathcal{X}^{ \pm}$and $\mathrm{X}^{ \pm}$binding energies is the signature of the fractional charge of the IQL

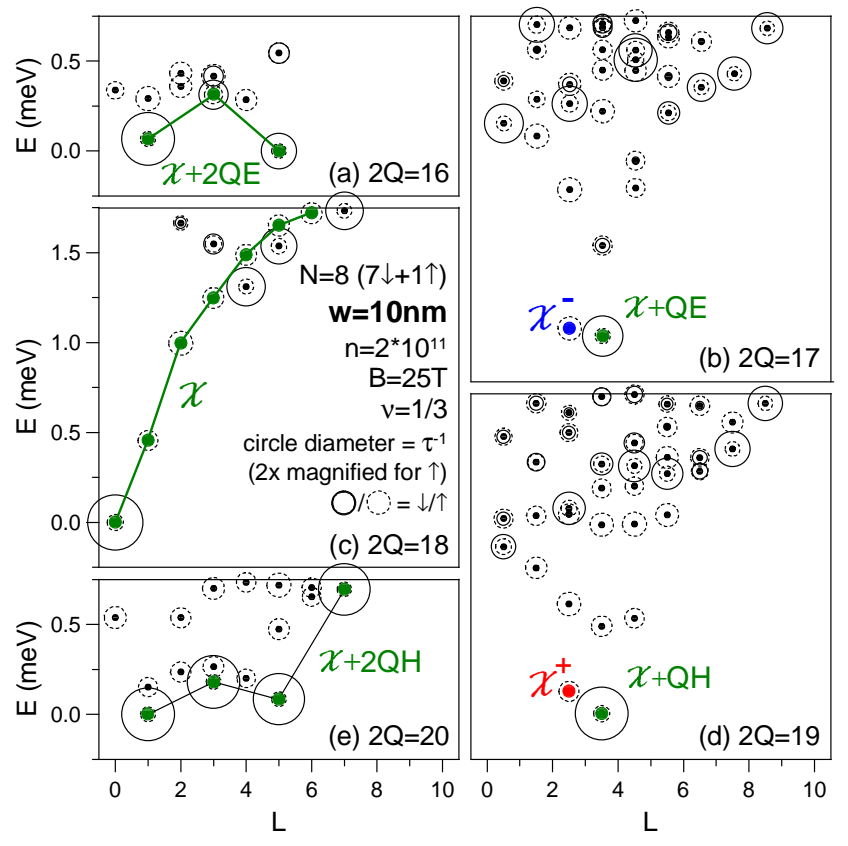

FIG. 4: (color online) Excitation spectra similar to Fig. 1 but for the $8 e+h$ systems with one reversed-spin electron and $w=10 \mathrm{~nm} . \tau^{-1}$ is separate for spin- $\downarrow$ and $\uparrow$ recombination.

excitations - directly observable as splittings in PL.

The QX's are defined through a sequence of gedanken processes: (i) trion binding: $2 e+h \rightarrow \mathrm{X}^{-}$, (ii) Laughlin correlation: $\mathrm{X}^{-} \rightarrow \mathcal{X}^{-}$, (iii) $\mathrm{QH}$ capture: $\mathcal{X}^{-} \rightarrow \mathcal{X} / \mathcal{X}^{+}$. Hence, $\mathcal{X}$ and $\mathcal{X}^{ \pm}$are the same $\mathrm{X}^{-}$, but differently separated from the surrounding electrons. This is evident in $e^{-} h$ pair-distribution functions (PDF's) in Fig. 3 Integration of $g(r)$ confirms fractional electron charge $-\frac{4}{3} e$, $-e$, and $-\frac{2}{3} e$ bound to the hole in the $\mathcal{X}^{-}, \mathcal{X}$, and $\mathcal{X}^{+}$.

Let us now turn to spin-unpolarized spectra, in search of QX's formed from the $\mathrm{X}_{\mathrm{s}}^{-}$. Its binding depends on LL mixing, so we used the following approximation. In the calculation of Coulomb matrix elements, the highest Haldane $e-e$ pseudopotential, $V_{0}$, was reduced by $10 \%$. This only affects interactions in the trion, and induces an (85\% accurate) $\mathrm{X}_{\mathrm{s}}^{-}$GS in the lowest LL. It has correct $g_{e h}$ and $g_{e e}$ PDF's, which determine coupling to the 2DEG.

From the analysis of $\tau^{-1}$ and $L$ we found $\mathcal{X}_{\mathrm{s}}$ and $\mathcal{X}_{\mathrm{s}}^{ \pm}$ in the spectra in Fig. [4 In contrast with Fig. [1 charged $\mathrm{QX}_{\mathrm{s}}$ 's are the excited states at $2 Q=17$ and 19 . Also at $2 Q=16$ and 20, the multiplicative states with an $\mathcal{X}_{\mathrm{s}}$ and two QP's lie below the $\mathcal{X}_{\mathrm{s}}^{-}-\mathrm{QE}$ and $\mathcal{X}_{\mathrm{s}}^{+}-\mathrm{QH}$ pairs.

This opposite behavior results from the $\mathrm{X}_{\mathrm{s}}^{-}$having different charge distribution than the $\mathrm{X}_{\mathrm{t}}^{-}$. It has little effect on its Laughlin correlation with the electrons, but affects its interaction with the QP's. Indeed, the $\mathcal{X}_{\mathrm{s}}$ dispersion in Fig. प(c) indicates stronger $\mathcal{X}_{\mathrm{s}}^{-}-\mathrm{QH}$ attraction.

We compare $\Delta^{0} \sim 2 \mathrm{meV}$ with $\Delta_{\mathrm{IQL}}=2.02 \mathrm{meV}$ using Eq. (1) to find that $\Delta^{-}$is very small or even negative, as in Fig. 田(b). Hence, even in the absence of free QH's, 
the $\mathcal{X}_{\mathrm{s}}^{-}$is unstable toward creation of a $\mathrm{QE}-\mathrm{QH}$ pair. Similarly, negative $\Delta^{+}$in Fig. प(d) implies instability of the $\mathcal{X}_{\mathrm{s}}^{+}$. As a result, the neutral $\mathcal{X}_{\mathrm{s}}$ is the most strongly bound state regardless of the presence of QE's or QH's.

This may add a continuous peak for the $w=20 \mathrm{~nm}$ well [see Fig. 2(b)], but precludes PL discontinuity in narrow wells with a strong $\mathrm{X}_{\mathrm{s}}^{-}$GS. The $\mathcal{X}_{\mathrm{s}}$ peak splits into a $\sigma_{ \pm}$doublet due to spin- $\downarrow$ and $\uparrow$ recombination involving either QE's or "reversed-spin" $\mathrm{QE}_{\mathrm{R}}$ 's [28], but temperature-activated emission at $k>0$ is not expected.

The QX idea can be extended to other IQL's (e.g., $\nu=\frac{2}{3}$ or $\frac{2}{5}$ ). However, different behavior of $\mathrm{QX}_{\mathrm{t}}$ 's and $\mathrm{QX}_{\mathrm{s}}$ 's at $\nu=\frac{1}{3}$ is an example that PL discontinuity is not guaranteed. Via Eq. (1), it is governed by sample- and $\nu$-dependent $\Delta_{\text {IQL }}$ and $\Delta^{0}$ which must be recalculated.

In summary, we have studied anomalies in PL of the IQL's in the regime of small charge separation. The emission spectrum is due to recombination of QX's formed from trions immersed in a 2DEG with Laughlin correlations. In spin-polarized systems, the neutral QX is equivalent to a nearly decoupled exciton at $k=0$, and its suppressed dispersion results from reduced charge of the constituents. The positive and negative spin-polarized QX's have fractional charge of one IQL QP. A spin-flip QX formed from a singlet trion was also found, with a steeper dispersion that prevents its charging by the IQL QP's. Featureless PL of the IQL in narrow $(10 \mathrm{~nm})$ wells and anomalies predicted for wider $(20 \mathrm{~nm})$ wells agree qualitatively with experiments.

We thank M. Potemski and P. Hawrylak for helpful discussions and sharing their results prior to publication. Work supported by grants DE-FG 02-97ER45657 of US DOE and 2P03B02424 of Polish MENiS.

[1] R. B. Laughlin, Phys. Rev. Lett. 50, 1395 (1983).

[2] D. C. Tsui, H. L. Störmer, and A. C. Gossard, Phys. Rev. Lett. 48, 1559 (1982).

[3] L. Saminadayar, D. C. Glattli, Y. Jin, and B. Etienne, Phys. Rev. Lett. 79, 2526 (1997); R. De-Picciotto, M. Reznikov, M. Heiblum, V. Umansky, G. Bunin, and D. Mahalu, Nature 389, 162 (1997).

[4] D. Heiman, B. B. Goldberg, A. Pinczuk, C. W. Tu, A. C. Gossard, and J. H. English, Phys. Rev. Lett. 61, 605 (1988).

[5] B. B. Goldberg, D. Heiman, A. Pinczuk, L. N. Pfeiffer, and K. West, Phys. Rev. Lett. 65, 641 (1990).

[6] A. J. Turberfield, S. R. Haynes, P. A. Wright, R. A. Ford, R. G. Clark, J. F. Ryan, J. J. Harris, and C. T. Foxon, Phys. Rev. Lett. 65, 637 (1990).

[7] F. Plentz, D. Heiman, A. Pinczuk, L. N. Pfeiffer, and K. W. West, Surface Sci. 361-362, 30 (1996); Solid State Commun. 101, 103 (1997).

[8] M. Byszewski, B. Chwalisz, D. K. Maude, M. L. Sadowski, M. Potemski, S. Studenikin, D. G. Austing, A. S. Sachrajda, P. Hawrylak, T. Saku, and Y. Hirayama,
SemiMag-16, Tallahassee, USA, August 2-6, 2004 (unpublished); private communication.

[9] G. Yusa, H. Shtrikman, and I. Bar-Joseph, Phys. Rev. Lett. 87, 216402 (2001).

[10] C. Schüller, K.-B. Broocks, P. Schröter, Ch. Heyn, D. Heitmann, M. Bichler, W. Wegscheider, T. Chakraborty, and V. M. Apalkov, Phys. Rev. Lett. 91, 116403 (2003).

[11] I. V. Kukushkin, R. J. Haug, K. von Klitzing, and K. Ploog, Phys. Rev. Lett. 72, 736 (1994); theory: V. M. Apalkov and E. I. Rashba, JETP Lett. 53, 442 (1991).

[12] A. Pinczuk, B. S. Dennis, L. N. Pfeiffer, and K. West, Phys. Rev. Lett. 70, 3983 (1993).

[13] V. M. Apalkov and E. I. Rashba, Phys. Rev. B 46, 1628 (1992); ibid. 48, R18312 (1993); J. Zang and J. Birman, ibid. 51, 5574 (1995).

[14] V. M. Apalkov, F. G. Pikus, and E. I. Rashba, Phys. Rev. B 52, 6111 (1995).

[15] E. I. Rashba and M. E. Portnoi, Phys. Rev. Lett. 70, 3315 (1993); M. E. Portnoi and E. I. Rashba, Phys. Rev. B 54, 13791 (1996); D. G. W. Parfitt and M. E. Portnoi, ibid. 68, 035306 (2003).

[16] X. M. Chen and J. J. Quinn, Phys. Rev. Lett. 70, 2130 (1993); A. Wójs and J. J. Quinn, Phys. Rev. B 63, 045303 (2001); ibid. 63, 045304 (2001).

[17] B. I. Halperin, Phys. Rev. Lett. 52, 1583 (1984).

[18] F. D. M. Haldane, Phys. Rev. Lett. 51, 605 (1983).

[19] J. K. Jain, Phys. Rev. Lett. 63, 199 (1989).

[20] I. V. Lerner and Yu. E. Lozovik, Sov. Phys. JETP 53, 763 (1981); A. B. Dzyubenko and Yu. E. Lozovik, Sov. Phys. Solid State 25, 874 (1983); D. Paquet, T. M. Rice, and K. Ueda, Phys. Rev. B 32, 5208 (1985); A. H. MacDonald and E. H. Rezayi, ibid. 42, R3224 (1990); A. H. MacDonald, E. H. Rezayi, and D. Keller, Phys. Rev. Lett. 68, 1939 (1992).

[21] K. Kheng, R. T. Cox, Y. Merle d'Aubigne, F. Bassani, K. Saminadayar, and S. Tatarenko, Phys. Rev. Lett. 71, 1752 (1993); H. Buhmann, L. Mansouri, J. Wang, P. H. Beton, N. Mori, M. Heini, and M. Potemski, Phys. Rev. B 51, R7969 (1995); G. Finkelstein, H. Shtrikman, and I. Bar-Joseph, ibid. 53, R10425 (1996); T. Vanhoucke, M. Hayne, M. Henini, and V. V. Moshchalkov, ibid. 65, 233305 (2002); B. M Ashkinadze, E. Linder, E. Cohen, A. B. Dzyubenko, and L.N. Pfeiffer, ibid. 69, 115303 (2004); G. V. Astakhov, D. R. Yakovlev, V. V. Rudenkov, P. C. M. Christianen, T. Barrick, S. A. Crooker, A. B. Dzyubenko, W. Ossau, J. C. Maan, G. Karczewski, and T. Wojtowicz, ibid. 71, 201312 (2005).

[22] A. Wójs and P. Hawrylak, Phys. Rev. B 51, 10880 (1995); J. J. Palacios, D. Yoshioka, and A. H. MacDonald, ibid. 54, R2296 (1996); A. B. Dzyubenko and A. Y. Sivachenko, Phys. Rev. Lett. 84, 4429 (2000).

[23] B. Stebe and A. Ainane, Superlatt. Microstruct. 5, 545 (1989); D. M. Whittaker and A. J. Shields, Phys. Rev. B 56, 15185 (1997); C. Riva, F. M. Peeters, and K. Varga, ibid. 63, 115302 (2001).

[24] A. Wójs, J. J. Quinn, and P. Hawrylak, Phys. Rev. B 62, $4630(2000)$.

[25] A. Wójs, I. Szlufarska, K. S. Yi, and J. J. Quinn, Phys. Rev. B 60, R11273 (1999); A. Wójs, P. Hawrylak, and J. J. Quinn, ibid. 60, 11661 (1999).

[26] I. H. Tan, G. L. Snider, L. D. Chang, and E. L. Hu, J. Appl. Phys. 68, 4071 (1990).

[27] F. D. M. Haldane, in The Quantum Hall Effect, edited by R. E. Prange and S. M. Girvin, (Springer-Verlag, New 
York, 1987), chapter 8, pp. 303-352.

[28] E. H. Rezayi, Phys. Rev. B 36, 5454 (1987). 